\title{
BMJ Open Protocol for the ROBUST (Registry Of type $B$ aortic dissection with the Utility of STent graft) study: an ambispective, multicentre, open cohort study
}

Dan Rong, ${ }^{1}$ Yangyang Ge, ${ }^{1}$ Yan Xue, ${ }^{2}$ Feng Liu, ${ }^{1,3}$ Kai Lu, ${ }^{1,4}$ Peng Liu, ${ }^{1,5}$ Lei Zhang, ${ }^{3}$ Xiaohu Ge, ${ }^{6}$ Jianhang Miu, ${ }^{7}$ Linkun Zhong, ${ }^{7}$ Weidong Fan, ${ }^{8}$ Hongpeng Zhang, ${ }^{1}$ Xin Jia, ${ }^{1}$ Xiaohui Ma, ${ }^{1}$ Jiang Xiong, ${ }^{1}$ Xiaoping Liu, ${ }^{1}$ Wei Guo ${ }^{1}$

To cite: Rong D, Ge Y, Xue Y, et al. Protocol for the ROBUST (Registry Of type B aortic dissection with the Utility of STent graft) study: an ambispective, multicentre, open cohort study. BMJ Open 2017;7:e019317. doi:10.1136/ bmjopen-2017-019317

- Prepublication history for this paper is available online. To view these files, please visit the journal online (http://dx.doi. org/10.1136/bmjopen-2017019317).

DR and YG contributed equally.

Received 25 August 2017 Revised 25 0ctober 2017 Accepted 26 October 2017

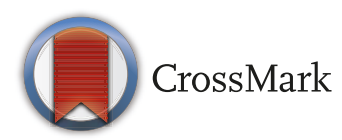

For numbered affiliations see end of article.

Correspondence to

Professor Wei Guo;

guowei@301hospital.com.cn

\section{ABSTRACT}

Introduction Thoracic endovascular aortic repair (TEVAR) is widely used for type B aortic dissection, although with satisfactory outcome in a limited proportion of patients. To better inform patient prognostication, the Registry of type $B$ aortic dissection with the Utility of STent graft (ROBUST) study aims to identify imaging-based predictors of postTEVAR adverse outcomes up to 10-year follow-up. Methods and analysis ROBUST is designed as an ambispective, multicentre, open cohort study. All patients undergoing TEVAR from 1 January 2008 to 1 July 2027 at participating centres will be invited to join the study. It is conservatively estimated that over 2000 patients will join the study. Data on demographics, disease history, procedural details, imaging features and follow-up will be collected after discharge. Cox proportional-hazards analysis will be used to identify independent predictors of primary outcomes. Stratification analysis will be performed to identify which subgroup of patients would benefit the most from TEVAR.

Ethics and dissemination The protocol has been approved by the ethics committee of the coordinating centre. Findings will be disseminated in professional peer-reviewed journals to promote understanding of the rehabilitation process.

Trial registration number ChiCTR-P0C-17011726; Preresults.

\section{INTRODUCTION}

Aortic dissection, first described several centuries ago, ${ }^{12}$ remained a fatally catastrophic vascular event until the 1950s when pertinent surgical interventions were introduced. ${ }^{3}$ Surgical repair of aortic dissection saved many lives, but its high perioperative mortality left room for improvement. ${ }^{4}$ Developments in medical devices and less invasive techniques led to significantly lower perioperative mortality and morbidity rates thanks to the introduction in 1999 of stent implantation to treat aortic dissection. ${ }^{5-7}$ Currently, thoracic endovascular aortic repair (TEVAR)
Strengths and limitations of this study

- ROBUST (Registry Of type B aortic dissection with the Utility of STent graft) is an ambispective, multicentre, open cohort study with up to 10 -year follow-up in a real-world setting.

- This is the first registry study mainly focusing on imaging features that affect the outcomes of patients after thoracic endovascular aortic repair.

- Clinical intervention will not be influenced because of the observational nature of the study.

- There might be a relatively high number of participants lost to follow-up over the 10-year follow-up period.

No control group will be included for comparison.

is recommended as first-line treatment for complicated type $\mathrm{B}$ aortic dissection (TBAD). ${ }^{8}$

Aortic dissection entails the separation of aortic wall layers secondary to bleeding within and along the aortic wall through an intimal tear. Aortic dissection is most commonly classified based on anatomical features using the Stanford classification, with type A dissection involving the ascending aorta regardless of primary entry tear site and type $\mathrm{B}$ dissection not involving it. ${ }^{9}$ In hospital-based studies in Western populations, the incidence of aortic dissection is 3-5 cases per 100000 people per year, which is probably an underestimation because of omission of preadmission deaths. ${ }^{10-12}$ In population-based studies, aortic dissection incidence is higher, namely 15 cases in the Western population and 43 cases in Asians per 100000 per year, maybe reflecting a higher prevalence of hypertension among Asians. ${ }^{13-15}$ The incidence can be even higher in the elderly population. In published studies, $20 \%-30 \%$ of aortic dissections are type B. ${ }^{1116}$ 
In TEVAR, stents are used to cover the primary entry tear and to support the collapsed intima. After stent implantation, blood flow is redirected towards the true lumen, thereby alleviating malperfusion of branch arteries, whereas entry tear coverage lowers the risk for dissection aneurysm by reducing false lumen pressure. The latter advantages translate into improved safety and efficacy. In the International Registry of Aortic Dissection, inhospital mortality was significantly lower for patients with TBAD treated with TEVAR versus surgically (11 patients $(10.8 \%)$ vs 26 patients $(23 \%))$, death rate at 5 years was significantly lower for patients who underwent endovascular treatment versus medical management $(15.5 \%$ vs $29.0 \%$, respectively), and moreover, descending aorta diameter at 5 years was significantly smaller after stent graft versus medical therapy alone (median, 4.2 (IQR $3.8-5.2) \mathrm{cm}$ vs4.6 (3.6-5.5) cm, respectively; $\mathrm{P}=0.034) .{ }^{16} 17$

Researchers also have investigated the prognosis of TBAD. Syncope, hypotensive or shock, diameter $\geq 55 \mathrm{~mm}$ and periaortic haematoma have been validated as risk factors for inhospital mortality. ${ }^{18}$ In clinical practice, not all patients who have undergone TEVAR show favourable aortic remodelling, and in addition to classic prognostic factors, such as the occurrence of complications (spinal ischaemia, mesenteric ischaemia, acute renal failure, hypotension and limb ischaemia) and severe symptoms (hypotension or shock, syncope, refractory hypertension and abdominal pain), imaging features have drawn increasing attention. However, studies involving imaging mostly have focused on common characteristics, such as aortic diameter, branch vessel involvement, periaortic haematoma and status of false lumen thrombosis. ${ }^{19-22}$ More studies on both clinical factors and imaging features specifically are warranted.

While previous studies have provided insight into prognosis after TEVAR, ${ }^{23-25}$ the Registry Of type B aortic dissection with the Utility of STent graft (ROBUST) will evaluate the evolving use and outcomes of TEVAR in a real-world setting. Furthermore, the registry will compare patients in different risk categories and may help identify which subgroup of patients would benefit the most from TEVAR.

\section{OBJECTIVE}

The primary objective of the registry is to identify predisposing risk factors and prognostic factors for adverse events in a real-world setting. Primary endpoint in present study is dissection aneurysm formation (defined as $>5 \mathrm{~mm}$ increase in maximum aortic diameter during a follow-up time point compared with the measurement at preoperative examination or a maximum aortic diameter $>55 \mathrm{~mm}$ ). Secondary endpoint is a composite endpoint including aortic-related death, endoleaks, retrograde type A aortic dissection, stent graft-related new entry, aortic rupture and impending rupture.

The secondary objectives are to: (1) generate more accurate indications for TEVAR by comparing patients in different risk-stratified subgroups, (2) assess the perioperative and long-term clinical outcomes of patients who have undergone and who are undergoing TEVAR and (3) assess the perioperative and long-term outcomes of patients after different types of reinterventions.

\section{METHODS \\ Study design}

The ROBUST study is an ambispective, multicentre, open cohort study. Participation is entirely voluntary, and the data will be collected after discharge. We plan to recruit consecutive patients after TEVAR from 2008 to 2027. After enrolment, all patients will be advised to undergo CT angiography (CTA) examination at 3, 6 and 12 months after intervention and yearly thereafter. We will acquire the clinical information from electronic patient databases and the CTA Digital Imaging and Communication in Medicine (DICOM) files from radiology department databases.

This registry is designed and initiated by the Department of Vascular and Endovascular Surgery, Chinese PLA General Hospital. To increase generalisability of study results, we invited four additional participating centres across China with enough experience to join this study, that is, each performing over 30 TEVAR yearly. The ROBUST study is an open project, and we welcome institutions fulfilling the specified requirements to join the study during the recruitment phase.

\section{Participants}

We will screen all patients who underwent TEVAR from 1 January 2008 to 1 July 2017 and will invite by phone patients with at least one follow-up time point to join our study. All patients admitted to hospitals for TEVAR after 1 July 2017 will be included in our study regardless of age, gender and underlying disease to reflect real-world practice. Recruitment is non-competitive and will not influence clinical practice.

\section{Criteria}

Inclusion criteria

1. Patients who have undergone and who will undergo TEVAR for TBAD.

2. Patients who agree to participate in the study.

\section{Exclusion criteria}

1. Patients for whom CTA would not be advisable (pregnant or preparing for pregnancy, lactating, with chronic renal failure or history of hypersensitivity to iodinated contrast media).

2. Patients lacking a preoperative CTA file in DICOM format or with images that are not amenable to reconstruction.

\section{Data collection}

A standard case report form (CRF) was designed at the beginning of our study. It will be used to obtain demographic information, disease history, surgical details, 
imaging features and outcome events of participants. Data will be primarily recorded on paper CRFs, then two researchers will input the CRFs into an electronic database simultaneously in case of typing errors.

\section{Data elements}

To address the objectives, we meticulously designed the CRF to collect the necessary information. The CRT consists of four parts, and the 184 variables compiled within the ROBUST study will include the following:

1. Demographics and disease history: age, gender, height, weight, tobacco consumption, chest or back pain, unconsciousness, breathlessness, hypotension, drinking habits, diabetes mellitus, hypertension, dyslipidaemia, ischaemic stroke, coronary artery disease, carotid stenosis and chronic obstructive pulmonary disease.

2. Surgical information: operation indication, American Society of Anesthesiologists physical status class, brand of stent graft, number of stent grafts, status of left subclavian artery (covered, uncovered or reconstructed), status of left common carotid artery (covered, uncovered or reconstructed), blood loss, contrast medium volume, type I endoleak during procedure, use of cerebrospinal fluid drainage and conversion to open surgery.

3. Preoperative imaging features: periaortic haematoma, impending rupture, pleural effusion, dissection length, primary tear location, branch arteries involvement, distal outflow track status, false lumen thrombosis status, diameter of aorta, true lumen and false lumen at different levels and volume of aorta, true lumen and false lumen.

4. Outcomes: periaortic haematoma, impending rupture, pleural effusion, proximal endoleak, stent graft-induced new entry, retrograde dissection, new re-entry tear in lower thoracic aorta, number of intimal tears, patency of left subclavian artery, patency of left vertebral artery, branch arteries involvement, false lumen thrombosis status of stent graft-covered segment, diameter of aorta, true lumen and false lumen at different levels, volume of aorta, true lumen and false lumen, ischaemic stroke, myocardial infarction, arterial embolism in lower limbs, paraplegia and death.

\section{Sample size and statistical analysis}

Previous studies pertaining to the effect estimation of a certain risk factor for the post-TEVAR aortic remodelling are limited. According to the results of our two previously published studies focusing on the association of the preoperative thoracic false lumen branches and the thoracic aortic enlargement after TEVAR, the point estimation of coefficient of the preoperative thoracic false lumen branches is 0.37 (95\% CI 0.18 to 0.57 ) for the association with the risk of thoracic aortic enlargement distal to stent graft and 1.17 (95\% CI 0.20 to 2.14) for the association with the risk of thoracic aortic enlargement along the stent graft. ${ }^{26}{ }^{27}$ Furthermore, the $\mathrm{R}^{2}$ of the preoperative thoracic false lumen branches with other covariates ranges from 0.254 to 0.301 . Additionally, our previous study and the Study for the Treatment of complicated Type B Aortic Dissection using Endoluminal repair (STABLE) reported that the incidence of thoracic aortic enlargement at 24 months after surgery ranges from $24 \%$ to $26 \%$. Based on these data, we conservatively used effect size of $0.18, \mathrm{R}^{2}$ of 0.35 and anticipated event rate of $20 \%$ to calculate the sample size. A minimum sample size of 93 patients will be included to achieve $80 \%$ power at a 0.05 significance level. However, the estimated sample size should be interpreted carefully, since the adopted data are derived from the study regarding the thoracic aortic remodelling rather than from the database focusing on aortic remodelling along both thoracic and abdominal aorta. From 2008 to 2017, more than 500 patients with TBAD have received endovascular repair in our hospital. More than 50 patients in our hospital and 30 patients in other four participating centres receive TEVAR every year. We will recruit patients consecutively from 2017 to 2027. A total number of 2000 patients are conservatively estimated to join the cohort, which is well exceed 93.

The results will be presented as frequencies and percentages for categorical variables and as means and SD for continuous variables. The different outcomes will be compared with the use of the $\mathrm{X}^{2}$ test for categorical variables and the Mann-Whitney test for continuous variables. Association between variables and outcomes first will be assessed by univariate regression analysis. After reviewing the variables for clinical significance, a subsequent Cox proportional-hazards analysis will be used to identify independent predictors of primary outcomes. Stratification analysis will be performed to identify which subgroup of patients would benefit the most from TEVAR.

\section{Ethics and dissemination}

The protocol has been approved by the ethics committee of the coordinating centre. This study was designed as an ambispective, observational registry, and the information will be collected after discharge. Therefore, clinical practice will not be influenced by the study. For patients admitted to hospitals for TEVAR before 1 July 2017, we will call them for verbal agreement. Signed informed consent will be obtained from patients admitted to hospitals after 1 July 2017 . The present study will be conducted in accordance with the provisions of the Declaration of Helsinki.

As soon as we fill out the CRFs, we will transfer them to an electronic database which will be stored both in a cloud environment and in a hard disk. Result dissemination is expected to commence in 2018 in professional peer-reviewed journals to promote understanding of the rehabilitation process.

Author affiliations

${ }^{1}$ Department of Vascular and Endovascular Surgery, Chinese PLA General Hospital, Beijing, China 
${ }^{2}$ Department of Cardiovascular Surgery, General Hospital of Armed Police Forces, Beijing, China

${ }^{3}$ Department of Vascular and Endovascular Surgery, The First Hospital of Hebei Medical University, Shijiazhuang, China

${ }^{4}$ Department of Vascular Surgery, Daqing Oil General Hospital, Daqing, China ${ }^{5}$ Department of Vascular Surgery, Affiliated Hospital of Jining Medical University, Jining, China

${ }^{6}$ Department of Vascular Surgery, People's Hospital of Xinjiang Uygur Autonomous Region, Urumchi, China

${ }^{7}$ Department of General Surgery, Zhongshan People's Hospital, Zhongshan, China

${ }^{8}$ Department of Cardiology, Henan Provincial Chest Hospital, Zhengzhou, China

Acknowledgements We greatly thank colleagues from participating centres for their contribution to this study, laotie shuangji 666.

Contributors WG, XL, LZha, XG, JM and WF planned the study. YG, XJ, JX, XM and $\mathrm{HZ}$ designed the study. $Y X, K L$ and $P L$ provided ethical support. DR and $Y G$ registered the study, wrote the study protocol and revised the manuscript. YG, DR, FL and LZho developed the CRF.

Competing interests None declared.

Patient consent Obtained.

Ethics approval IRB of Chinese PLA General Hospital.

Provenance and peer review Not commissioned; externally peer reviewed.

Data sharing statement The ROBUST study is an open project. We will share our data after arrangement. We welcome institutions experienced with TEVAR to join the study. Anyone interested in our research can download the data from the website: huiyihuiying.com or contact us directly.

Open Access This is an Open Access article distributed in accordance with the Creative Commons Attribution Non Commercial (CC BY-NC 4.0) license, which permits others to distribute, remix, adapt, build upon this work non-commercially, and license their derivative works on different terms, provided the original work is properly cited and the use is non-commercial. See: http://creativecommons.org/ licenses/by-nc/4.0/

(c) Article author(s) (or their employer(s) unless otherwise stated in the text of the article) 2017. All rights reserved. No commercial use is permitted unless otherwise expressly granted.

\section{REFERENCES}

1. Acierno L. The history of cardiology. New York: Parthenon Publishing Group, 1994

2. Hagan PG, Nienaber CA, Isselbacher EM, et al. The international registry of acute aortic dissection (IRAD): new insights into an old disease. JAMA 2000;283:897-903.

3. Warden HE, COHEN M, READ RC, et al. Controlled cross circulation for open intracardiac surgery: physiologic studies and results of creation and closure of ventricular septal defects. $J$ Thorac Surg 1954;28:331-41.

4. Fattori R, Tsai TT, Myrmel T, et al. Complicated acute type B dissection: is surgery still the best option?: a report from the international registry of acute aortic dissection. JACC Cardiovasc Interv 2008;1:395-402.

5. Dake MD, Kato N, Mitchell RS, et al. Endovascular stent-graft placement for the treatment of acute aortic dissection. N Engl J Med 1999;340:1546-52.

6. Nienaber CA, Fattori R, Lund G, et al. Nonsurgical reconstruction of thoracic aortic dissection by stent-graft placement. N Engl J Med 1999;340:1539-45.
7. Zeeshan A, Woo EY, Bavaria JE, et al. Thoracic endovascular aortic repair for acute complicated type $B$ aortic dissection: superiority relative to conventional open surgical and medical therapy. $J$ Thorac Cardiovasc Surg 2010;140:S109-15.

8. Nienaber CA, Clough RE, Sakalihasan N, et al. Aortic dissection. Nat Rev Dis Primers 2016;2:16053.

9. Daily PO, Trueblood HW, Stinson EB, et al. Management of acute aortic dissections. Ann Thorac Surg 1970;10:237-47.

10. Clouse WD, Hallett JW, Schaff HV, et al. Acute aortic dissection: population-based incidence compared with degenerative aortic aneurysm rupture. Mayo Clin Proc 2004;79:176-80.

11. Mészáros I, Mórocz J, Szlávi J, et al. Epidemiology and clinicopathology of aortic dissection. Chest 2000;117:1271-8.

12. Sato F, Kitamura T, Kongo M, et al. Newly diagnosed acute aortic dissection: characteristics, treatment modifications, and outcomes. Int Heart J 2005;46:1083-98.

13. Yu HY, Chen YS, Huang SC, et al. Late outcome of patients with aortic dissection: study of a national database. Eur $\mathrm{J}$ Cardiothorac Surg 2004;25:683-90.

14. Landenhed M, Engström G, Gottsäter A, et al. Risk profiles for aortic dissection and ruptured or surgically treated aneurysms: a prospective cohort study. J Am Heart Assoc 2015;4:e001513.

15. Yeh TY, Chen CY, Huang JW, et al. Epidemiology and medication utilization pattern of aortic dissection in taiwan: a population-based study. Medicine 2015;94:e1522.

16. Fattori R, Montgomery D, Lovato $\mathrm{L}$, et al. Survival after endovascular therapy in patients with type $\mathrm{b}$ aortic dissection. JACC Cardiovasc Interv 2013;6:876-82.

17. Booher AM, Isselbacher EM, Nienaber CA, et al. The IRAD classification system for characterizing survival after aortic dissection. Am J Med 2013;126:730.e19-24.

18. Tolenaar JL, Froehlich W, Jonker FH, et al. Predicting inhospital mortality in acute type B aortic dissection: evidence from international registry of acute aortic dissection. Circulation 2014;130(Suppl 1):S45-50.

19. Tsai TT, Evangelista A, Nienaber CA, et al. Partial thrombosis of the false lumen in patients with acute type B aortic dissection. N Engl J Med 2007;357:349-59.

20. Kim KM, Donayre CE, Reynolds TS, et al. Aortic remodeling, volumetric analysis, and clinical outcomes of endoluminal exclusion of acute complicated type B thoracic aortic dissections. J Vasc Surg 2011:54:316-25.

21. Watanabe $\mathrm{Y}$, Shimamura $\mathrm{K}$, Yoshida $\mathrm{T}$, et al. Aortic remodeling as a prognostic factor for late aortic events after thoracic endovascular aortic repair in type B aortic dissection with patent false lumen. $J$ Endovasc Ther 2014;21:517-25.

22. Booher AM, Isselbacher EM, Nienaber CA, et al. Ascending thoracic aorta dimension and outcomes in acute type B dissection (from the International Registry of Acute Aortic Dissection [IRAD]). Am J Cardiol 2011:107:315-20.

23. Shirali AS, Bischoff MS, Lin HM, et al. Predicting the risk for acute type $\mathrm{B}$ aortic dissection in hypertensive patients using anatomic variables. JACC Cardiovasc Imaging 2013;6:349-57.

24. Afifi RO, Sandhu HK, Leake SS, et al. Outcomes of patients with acute type B (DeBakey III) aortic dissection: a 13-year, single-center experience. Circulation 2015;132:748-54.

25. Pape LA, Awais M, Woznicki EM, et al. Presentation, diagnosis, and outcomes of acute aortic dissection: 17-year trends from the international registry of acute aortic dissection. J Am Coll Cardiol 2015;66:350-8.

26. Liu F, Ge YY, Guo W, et al. Preoperative thoracic false lumen branches are predictors of aortic enlargement after stent grafting for DeBakey Illb aortic dissection. J Thorac Cardiovasc Surg 2017. doi: 10.1016/j.jtcvs.2017.09.010. [Epub ahead of print 15 Sep 2017].

27. Ge YY, Guo W, Cheshire N, et al. Preoperative thoracic false lumen branches relate to aortic remodeling after thoracic endovascular aortic repair for DeBakey IIIb aortic dissection. J Vasc Surg 2017;65:659-68. 